\title{
COMMISSION 25: STELLAR PHOTOMETRY AND POLARIMETRY
}

\author{
(PHOTOMETRIE ET POLARIMETRIE STELLAIRES)
}

\author{
PRESIDENT: C. Sterken \\ VICE-PRESIDENT: A. Landolt \\ ORGANIZING COMMITTEE: S. Adelman, P. Bastien, M. Bessell, S. Fabrika, \\ R. Gilliland, I. Glass, J. Graham, H. Hensberge, P. Martinez, E.F. Milone, \\ K. Sekiguchi, P. Stetson, V. Straižys, A. Walker \& W.H. Warren
}

A vast number of papers relying on photometry and polarimetry to investigate variable stars, open and globular clusters, galaxies, quasars etc., is being produced (not to speak of large-scale surveys) and it is impossible to give an overview of these activities in the allotted space. Many of these results are described in the reports by other Commissions, so the present report will deal only with developments related to photometric techniques, i.e. instrumentation, photometric systems, etc.

\section{Photometric Instruments}

A CCD-based spectrophotometer for a new automated 0.5-m telescope at the Fairborn Observatory, (AZ) USA, is under construction and should see first light in Spring 2004. The Citadel ASTRA (Automated Spectrophotometric Telescope Research Associates) Telescope will permit observations of the primary spectrophotometric standard Vega, rapid measurements of the naked-eye stars, sufficient observing time to obtain photometric measurements of the nightly extinction, and still obtain high-quality observations of stars of about 10.5 magnitude in an hour. This multiplexed cross-dispersed instrument should produce highquality fluxes at least of 3300-9000 Angstroms with a resolution of 14 Angstroms in first and 7 Angstroms in second order and full wavelength coverage except for regions badly affected by telluric lines. For details, see http://www.citadel.edu/physics/astra/index.html.

A very useful source of instrumental expertise has been set up by Jaap Tinbergen: the Dictionary of Astronomical Instrumentation (http://www.strw.leidenuniv.nl/dai/) is a teach-yourself manual on the basic principles of astronomical instrumentation, from $\gamma$ rays to the longest radio waves. It refers to existing literature (paper and electronic), and introductory texts help the reader to understand the technical jargon. The tutorial is intended for astronomers, engineers and students.

\section{Photometric Systems}

\subsection{Asiago Database on Photometric Systems}

The Asiago Database on Photometric Systems (ADPS) project outlines a detailed overview of more than 200 photometric systems, including basic information and reference data and an extensive bibliography. A useful website http://ulisse.pd.astro.it/Astro/ADPS/ gives detailed information and historical references, passband data, response curves and a link to the General Catalogue of Photometric Data.

\subsection{Infrared Photometry: the IRWG System}

A detailed report on the developments of the photometric IRWG system is given by the Working Group on Infrared Astronomy, see further in these Transactions. 


\subsection{Photometry from Space}

Several space missions implementing photometry of some kind are planned for the near future: MOST (Microvariability and Oscillations of Stars; www.astro.ubc.ca/MOST/), MONS (Measuring Oscillations in Nearby Stars; http://www.obs .aau.dk/MSC/), COROT (COnvection ROtation and planetary Transits; http://www.astrsp-mrs.fr/projets/) and Eddington (http://sci.esa.int/home/eddington/). A major effort has been the designing of photometric systems (i.e. calibrated and multi-band) for space observations, specifically for the GAIA mission.

GAIA, ESA's cornerstone 6 mission with a launch not later than 2012, will scan the full sky during five years measuring all objects down to $V=20$ (about 1 billion objects, i.e. approx. $1 \%$ of the Galaxy). The main goal of the mission is the understanding of Galaxy composition, formation and evolution. Position, space velocity and photometry will be acquired for each source.

GAIA will measure all detected sources with a broad-band photometer implemented by 5 filter bands in the focal plane of the astrometric telescopes. The sources will also be measured with a medium-band photometer implemented by about 11 filter bands in the focal plane of the smaller Spectro telescope. Additionally, high/medium resolution spectra in the range 849-874 $\mathrm{nm}$ will be acquired mainly for radial velocity determination.

A Photometry Working Group was created with the main objective of designing optimal photometric system(s) to be implemented in the mission, taking into account astrophysical diagnostics across the broadest range of sources, chromatic effects on astrometry, sampling strategy, background determination and compression possibilities. The main activities of the PWG during the last years were:

- Broad- and medium-band photometry: Three broad-band and three medium-band proposals were elaborated for optimal stellar astrophysical parameters determination. The different medium-band proposals are being evaluated for a convergence to a single system, and the performances of the three proposals are being evaluated as a function of apparent magnitude and stellar population physical and kinematical properties. The determination of reddening, temperature, gravity and metallicity combining the data coming from all instruments on board GAIA is under study. The performances for multiple stars, peculiar stars, emission line stars and non-stellar objects (QSO, solar-system, galaxies) are being evaluated.

- Interstellar absorption: Methods to determine a 3-D map of the insterstellar absorption from GAIA photometry are ongoing. Complementarity of data coming from other missions is also under study.

- Automatic classification/parameterization algorithms: In cooperation with the Classification WG, minimum distance and neural network methods are under development for testing the capabilities of the present proposals and further automatic classification and parameterization of the one billion of observed objects.

- Photometry simulations: A web tool for simulation of GAIA photometric observations was developed and is available at http://gaia.am.ub.es/PWG/.

Three meetings in July 2001, November 2001 and July 2002 took place in Vilnius, Barcelona and Tartu, respectively. Proceedings of the Vilnius meeting were edited by V. Vansevicius et al. 2002 (Census of the Galaxy, repr. from ApSS Vol. 280). Reports of the other meetings are on http://astro.estec.esa.nl/GAIA and http://gaia.am.ub.es/PWG/

Acknowledgments. Contribution by Carme Jordi on GAIA is gratefully acknowledged. C. Sterken

President of the Commission 